\title{
Prediction of an Upper Limit for the Fraction of Interprotein Cross-Links in Large-Scale In Vivo
}

\section{Cross-Linking Studies}

Article

Andrew Keller ${ }^{1}$, Juan D. Chavez ${ }^{1}$, Kevin C. Felt ${ }^{1}$, and James E. Bruce ${ }^{1^{*}}$

${ }^{1}$ Department of Genome Sciences, University of Washington, Seattle, WA 98195 USA

jimbruce@uw.edu

\section{SUPPORTING INFORMATION}

- $\quad$ Figure S1: Effect of the PDB Structure Complex Size on Interprotein Fractions

- Estimation of Maximum Sample Interprotein Fraction by Central Limit Theorem

- Derivation of Equation 1

- Derivation of Equation 2

- Table S1: Comparison of PDB Interprotein Cross-Link Fractions Calculated using SASD versus Euclidean Maximum Distance

- Table S2: Effect of FDR on the Fraction of Interprotein Cross-Links

- Estimated Undercount of Interprotein Cross-Links 


\section{Effect of the PDB Structure Complex Size on Interprotein Fractions}

The effect of minimum number of PDB bound constituents, reflecting complex size, on the resulting mean fraction of interprotein cross-links is shown below in Figure S1.

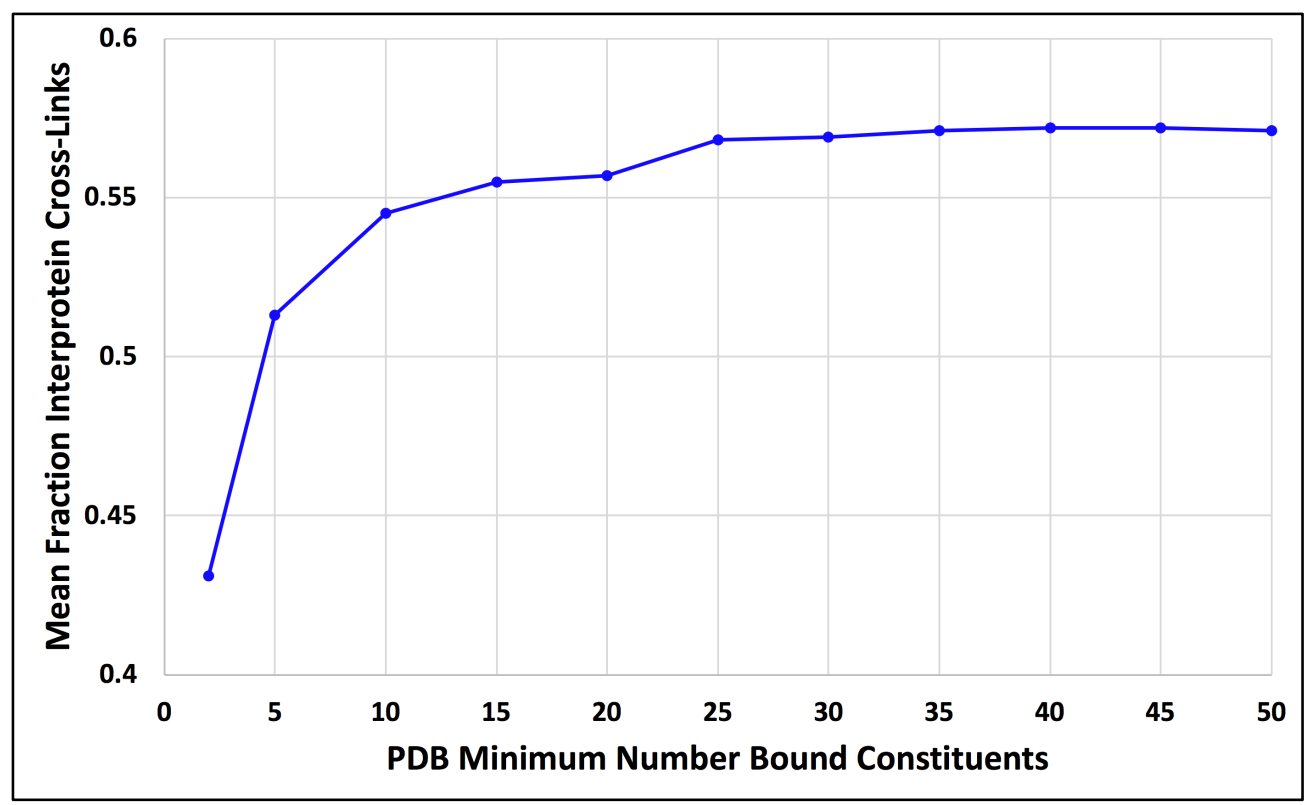

Figure S1. Mean fraction of interprotein cross-links computed among PDB structures with indicated minimum numbers of bound constituents.

It is evident that the mean fraction of interprotein cross-links plateaus for PDB files with a minimum number of 25 bound constituents, or greater.

\section{Estimation of Maximum Sample Interprotein Fraction by Central Limit Theorem}

If we assume the 1000 sample cross-links originate from a multitude of random large complexes represented in the distribution of interprotein fractions based on PDB structures with $\mathbf{2 5}$ or more bound constituents, then the likelihood that each cross-link is interprotein can be independently derived from that distribution. The resulting experiment samples are expected to have fractions of interprotein cross-links with a mean value equal to the PDB-based distribution mean, $\mu_{\xi}(0.568)$, and standard deviation equal to $\sigma_{\xi} / \sqrt{N}$, where $\sigma_{\xi}$ is the PDBbased distribution standard deviation (0.09) and $\mathrm{N}$ is the number of sample cross-links. According to the Central Limit Theorem, the distribution of sample interprotein fractions for datasets of 1000 cross-links is expected to be close to normal despite the original PDB-based distribution skewing to the right. Consequently $99 \%$ of samples with 1000 or more cross-links 
are expected to have a fraction of interprotein cross-links, $\xi$, no greater than three standard deviations above the mean fraction, i.e. $\mu_{\xi}+3 \sigma_{\xi} / \sqrt{N}$. A predicted maximum value for $\xi$ is thus calculated as:

$$
\xi_{\max } \approx \mu_{\xi}+3 \sigma_{\xi} / \sqrt{1000}=\mu_{\xi}+0.095 \sigma_{\xi}
$$

For the case of a $35 \AA$ spanning cross-linker with PDB-based distribution mean $\mu_{\xi}$ equal to 0.568 and standard deviation $\sigma_{\xi}$ equal to 0.09 , one obtains a predicted maximum fraction of interprotein cross-links, $\xi_{\max }$, equal to 0.58 . This is close to the estimated maximum value of 0.6 derived by Monte Carlo simulation of sample interprotein fractions.

\section{Derivation of Equation 1}

Assume in a dataset there are $N$ true positive cross-link results and $F$ false positive results. Also assume that the fraction of true positive cross-links that are interprotein is equal to $\xi_{0}$, while the fraction of false positives that are interprotein is 1 . Then the combined fraction of all results, both true and false positive, that are interprotein, $\xi$, is given by:

$$
\xi=\frac{\xi_{0} N+F}{N+F}
$$

Rearranging and solving for $N$ yields:

$$
N=\frac{(1-\xi) F}{\left(\xi-\xi_{0}\right)} \text { where } \xi>\xi_{0}
$$

Substituting this value for $N$ into the formula for FDR yields Equation 1:

$$
F D R=\frac{F}{N+F}=\frac{\xi-\xi_{0}}{1-\xi+\xi-\xi_{0}}=\frac{\xi-\xi_{0}}{1-\xi_{0}} \text { where } \xi>\xi_{0}
$$

More generally, if not all false positives are necessarily interprotein, but instead a fraction $f$ ranging between $\xi$ and 1, then Equation 1 becomes:

$$
F D R=\frac{\xi-\xi_{0}}{f-\xi_{0}} \text { where } f \geq \xi>\xi_{0}
$$




\section{Derivation of Equation 2}

Equation 1 can be extended to the case where an observed fraction of interprotein cross-links, $\xi$, is greater than the predicted maximum value, $\xi_{\text {max }}$, which in turn is greater than or equal to the actual fraction among true positives, $\xi_{0}$, i.e. where $\xi>\xi_{\max } \geq \xi_{0}$.

The observed interprotein fraction, $\xi$, is by definition no greater than 1 :

$$
1 \geq \xi
$$

Multiplying both sides of Equation S5 by the non-negative $\left(\xi_{\max }-\xi_{0}\right)$ yields:

$$
\xi_{\max }-\xi_{0} \geq \xi \xi_{\max }-\xi \xi_{0}
$$

Adding the term $\left(\xi+\xi_{0} \xi_{\max }-\xi \xi_{\max }-\xi_{\max }\right)$ to both sides of Equation $\mathrm{S6}$ gives:

$$
-\xi \xi_{\max }-\xi_{0}+\xi+\xi_{0} \xi_{\max } \geq-\xi_{\max }-\xi \xi_{0}+\xi+\xi_{0} \xi_{\max }
$$

Upon factoring both sides of Equation S7, one obtains:

$$
\left(\xi-\xi_{0}\right)\left(1-\xi_{\max }\right) \geq\left(\xi-\xi_{\max }\right)\left(1-\xi_{0}\right)
$$

Since $\xi_{0} \leq \xi_{\max }<\xi \leq 1$, it is true that $\xi_{\max }<1$ and $\xi_{0}<1$. Dividing both sides of Equation S8 by the non-negative non-zero product $\left(1-\xi_{\max }\right)\left(1-\xi_{0}\right)$ leads to:

$$
\frac{\xi-\xi_{0}}{1-\xi_{0}} \geq \frac{\xi-\xi_{\max }}{1-\xi_{\max }} \text { where } \xi>\xi_{\max } \geq \xi_{0}
$$

According to Equation 1, the first term of Equation $S 9$ is equal to FDR, assuming all false positives are interprotein. Upon substitution into Equation S9, this yields Equation 2:

$$
F D R \geq \frac{\xi-\xi_{\max }}{1-\xi_{\max }} \text { where } \xi>\xi_{\max } \geq \xi_{0}
$$

More generally, if not all false positives are necessarily interprotein, but instead a fraction $f$ ranging between $\xi$ and 1, then Equation 2 becomes:

$$
F D R \geq \frac{\xi-\xi_{\max }}{f-\xi_{\max }} \text { where } f \geq \xi>\xi_{\max } \geq \xi_{0}
$$




\section{Comparison of PDB Interprotein Cross-Link Fractions Calculated using SASD versus Euclidean}

Maximum Distance

Predicted fractions of interprotein cross-links were calculated for all lysine pairs within Jwalk SASD maximum distance ranging from 5 to $50 \AA$ for a subset of 1000 PDB files selected at random from those 18,676 used for distributions shown in Figure 1. These structures include a wide range of numbers of bound constituents, in similar proportion to the complete PDB file set: 130 have 10 or more constituents, 85 have 15 or more, and 62 have 25 or more. Interprotein fractions calculated using SASD were compared with those calculated for the same structure using Euclidean distance. Results of linear correlation are shown below in Table S1. The correlation improves with increasing maximum distance, likely due to the increased number of lysine pairs with which to compute an interprotein fraction. Also shown are the linear correlation slope and intercept, ideally equal to 1 and 0 , respectively, the average number of lysine pairs within maximum distance (Euclid or SASD) per structure, among which the interprotein fraction was calculated, and the number of PDB files contributing to the linear correlation.

\begin{tabular}{|c|c|c|c|c|c|c|}
\hline $\begin{array}{c}\text { Maximum } \\
\text { Inter-Lysine } \\
\text { Distance (Å) }\end{array}$ & \multirow{2}{*}{$\begin{array}{c}\text { Correlation SASD } \\
\text { versus Euclid }\end{array}$} & Slope & \multirow{2}{*}{ Intercept } & \multicolumn{2}{|c|}{$\begin{array}{c}\text { Average Number Lysine } \\
\text { Pairs per Structure }\end{array}$} & $\begin{array}{c}\text { Number } \\
\text { Structures } \\
\text { Compared }\end{array}$ \\
\cline { 4 - 6 } & & & & Euclid & SASD & 176 \\
\hline 5 & 0.84 & 0.92 & 0 & 31 & 26 & 689 \\
\hline 10 & 0.85 & 0.92 & 0 & 76 & 46 & 929 \\
\hline 15 & 0.91 & 0.95 & 0 & 250 & 100 & 988 \\
\hline 20 & 0.94 & 0.97 & 0 & 459 & 182 & 995 \\
\hline 25 & 0.96 & 0.98 & 0 & 754 & 292 & 1000 \\
\hline 30 & 0.97 & 0.98 & 0 & 1113 & 428 & 1000 \\
\hline 35 & 0.98 & 0.99 & 0 & 1524 & 590 & 1000 \\
\hline 40 & 0.98 & 0.99 & 0 & 1972 & 772 & 1000 \\
\hline 45 & 0.99 & 0.99 & 0 & 2458 & 973 & 1000 \\
\hline 50 & 0.99 & 1 & 0 & 2973 & 1185 & \\
\hline
\end{tabular}

Table S1. The correlation among calculated fraction of interprotein cross-links for lysine pairs within indicated maximal SASD versus Euclidean distance, for 1000 random PDB structure files contributing to the distributions of Figure 1.

\section{Effect of FDR on the Fraction of Interprotein Cross-Links}

Properties of the five datasets of non-redundant cross-links filtered for increasing decoyestimated FDR to assess its effect on the observed interprotein fraction, are shown below in 
Table S2. Indicating are their cross-linker, fraction of interprotein cross-links among decoy, low scoring (maximum Comet expect score 35 or greater), and true-positive (filtered at decoyestimated 0\% FDR) cross-link results. Also shown are their numbers of non-redundant crosslinks at $1 \%$ FDR, as estimated by XLinkProphet.

Decoy-estimated FDR followed Walzthoeni et al. ${ }^{1}$, whereby the number of target false positives is computed as the number of cross-links spanning a decoy at one end minus the number spanning a decoy at both ends. Since all datasets were searched with whole proteome databases, ranging from prokaryotic to eukaryotic, applying this formula for non-directional cross-link data should be a valid approximation, as discussed in Fischer and Rappsilber².

\begin{tabular}{|l|c|c|c|c|c|c|}
\hline \multicolumn{1}{|c|}{ Dataset } & Cross-Linker & $\begin{array}{c}\text { Interprotein } \\
\text { Fraction of } \\
\text { Decoy Cross- } \\
\text { Links }\end{array}$ & $\begin{array}{c}\text { Interprotein } \\
\text { Fraction of Low } \\
\text { Scoring Cross-Links } \\
\text { (Max Expect } \geq \mathbf{3 5} \text { ) }\end{array}$ & $\begin{array}{c}\text { Interprotein } \\
\text { Fraction of True } \\
\text { Positive Cross- } \\
\text { Links, } \xi_{0}\end{array}$ & $\begin{array}{c}\text { Number Non- } \\
\text { Redundant Cross- } \\
\text { Links at 1\% FDR }\end{array}$ & $\begin{array}{c}\text { Estimated } \\
\text { Target } \\
\text { Database Size }\end{array}$ \\
\hline Nuclear DSSO Mango & DSSO & 1 & 1 & 0.245 & 3928 & 705 \\
\hline Histone Protein Mixture & PIR & 1 & 1 & 0.402 & 371 & 58 \\
\hline HeLa ReACT & PIR & 1 & No Results & 0.198 & 2881 & 1032 \\
\hline E.coli Mango & PIR & 1 & 0.96 & 0.066 & 3895 & 40 \\
\hline Mouse Heart ReACT & PIR & 1 & No Results & 0.235 & 2461 & 3174 \\
\hline
\end{tabular}

Table S2. Cross-linked datasets filtered for increasing decoy-estimated FDR.

\section{Estimated Undercount of Interprotein Cross-Links}

On XLinkDB public datasets, there are 77 different proteins with unambiguous evidence for homo-dimerization (cross-link attached at both ends to the same lysine position in the protein), comprising just $3 \%$ of all cross-linked proteins. For presumed intraprotein cross-links identified for those proteins (i.e. with cross-links attached at each end to distinct lysine positions in the protein), approximately $1 / 3$ had Euclidean distances in the context of a modeled docked dimer structure within the expected $35 \AA$ distance. This is significantly less than the $86 \%$ of cross-links with intraprotein cross-link distances within $35 \AA$ in the context of the monomer structure. Nevertheless, these numbers suggest that as many as $3 \% * 1 / 3$, or just $1 \%$ of our presumed intraprotein cross-links, may also, or instead, be interprotein cross-links. In the unlikely extreme case that all cross-linked proteins are present in samples bound as homo-dimers, as 
many as $1 / 3$ of presumed intraprotein cross-links can instead be interprotein, significantly inflating observed dataset fractions of interprotein cross-links.

\section{References}

1. Walzthoeni, T.; Claassen, M.; Leitner, A.; Herzog, F.; Bohn, S.; Förster, F.; Beck, M.; Aebersold, R., False discovery rate estimation for cross-linked peptides identified by mass spectrometry. Nature Methods 2012, 9, 901.

2. Fischer, L.; Rappsilber, J., Quirks of Error Estimation in Cross-Linking/Mass Spectrometry. Anal Chem 2017, 89, (7), 3829-3833. 